O2E.4 EVALUATION OF EXPOSURE ASSESSMENT METHODS IN EPIDEMIOLOGICAL STUDIES: THE WELDING EXAMPLE

${ }^{1,2}$ Susan Peters ${ }^{*},{ }^{3}$ Jerome Lavoue, ${ }^{4}$ Marissa Baker, ${ }^{1}$ Hans Kromhout. ${ }^{1}$ Institute for Risk Assessment Sciences, Utrecht University, Utrecht, Netherlands; ${ }^{2}$ University Medical Centre Utrecht, Utrecht, Netherlands; ${ }^{3}$ University of Montreal, Montreal, Canada; ${ }^{4}$ University of Washington, Seattle, USA

\subsection{6/OEM-2019-EPI.55}

Exposure assessment quality is a fundamental consideration in the design and evaluation of observational studies. High quality exposure assessment is particularly relevant for outcomes with long latency, such as cancer, where detailed information on past exposures are often missing and must therefore be estimated.

For the IARC Monograph on welding, the exposure group provided an overview of assessment methods used in the key epidemiological studies. Strengths and weaknesses of each study were assessed, along with their potential effects on interpretation of risk estimates.

For the association between lung cancer and welding fume exposure, 9 cohort and 10 case-control studies were reviewed. For ocular melanoma and ultraviolet radiation (UVR) from welding, 7 case-control studies were reviewed. Quality criteria were: full occupational histories, and standardized, blinded and quantitative exposure assessment. Additional criteria for lung cancer: specifically assessing welding fumes and using information on welding tasks. For ocular melanoma: assessing artificial and solar radiation separately, taking into account eye burns, eye protection and welding type.

Exposure assessment of welding fumes by applying a 'welding-exposure matrix' $(n=2)$ or welding-specific questionnaires $(n=3)$ were considered highest quality, followed by case-by-case expert assessment $(n=5)$ or general job-exposure matrices (JEMs, $n=4)$. Job title alone was considered less informative $(n=5)$. For exposure to UVR, JEMs were most informative $(n=2)$, followed by self-reported eye burns and self-reported exposure from specific welding types $(n=2)$, although caution is advised regarding recall bias. Assessing welding fume exposure or ever exposure to welding arcs as proxy for UVR was considered less informative. For both exposures, ever versus never welder, or assessments based on data collected from proxies, were considered least informative.

The overall evaluation was that there is sufficient evidence in humans for the carcinogenicity of welding fumes and ultraviolet radiation from welding.

\section{O2E.5 CHALLENGES IN PESTICIDE EXPOSURE ASSESSMENT FOR EPIDEMIOLOGIC STUDIES}

Laura Beane Freeman*. Occupational and Environmental Epidemiology Branch, US National Cancer Institute, Rockville, USA

\subsection{6/OEM-2019-EPI.56}

Pesticides are used extensively worldwide to control weeds, insects and other pests, some of which have been linked to numerous adverse health outcomes, including cancer. Their ubiquity makes it imperative to conduct high quality epidemiologic studies of these chemicals for hazard identification and risk assessment. There are hundreds of pesticides and pesticide active ingredients registered for use worldwide. However, only a handful have been formally evaluated for carcinogenicity, partially due to the paucity of high quality studies with information on specific active ingredients. Some of the challenges related to assessing exposure to these chemicals are not unique, but there are some particular circumstances that make evaluation of pesticides particularly challenging. Although agricultural practices are changing in many parts of the world, pesticides have traditionally been applied on family farms or small workplaces with few employees. Many studies have relied on the self-report of pesticides; however, depending on the specific work practices, the workers applying the pesticides may not know what chemicals are being applied and may not be able to report the specific active ingredients directly. Other studies have inferred exposure to individual chemicals using a variety of methods, including individual reports of general pesticide application, the crops being grown and combining these data with auxiliary information, such as sales or registration records, application diaries or the use of local experts to assign probability of exposure. In addition to the specific active ingredient, the intensity of exposure is related to other factors, including those associated with application methods and the use of personal protective equipment. Pesticides are often applied in combination with one another, or in mixtures and applicators may apply or be exposed to multiple chemicals through a growing season. Despite these, and other challenges, high quality exposure assessment is critical to studies of these important chemicals.

\section{O2E.6 DEFINING SHIFT WORK EXPOSURE IN EPIDEMIOLOGICAL STUDIES OF CANCER: WHERE HAVE WE BEEN, AND WHAT COMES NEXT?}

Amy Hall* . International Agency for Research on Cancer, Lyon, France

\subsection{6/OEM-2019-EPI.57}

In the epidemiological context, 'exposure' to shift work encompasses a variety of social, workplace, and individual characteristics. This complexity lends itself to coarse exposure assessment and assignment, which increases misclassification error and often reduces contrast between comparison groups. In early epidemiological studies of shift work, broad exposure assignment, such as 'day' versus 'night' worker, was commonly used; a technique that ignored various characteristics (e.g., shift intensity, timing, and work history) with potential impacts on long-term health risks.

In 2007, The International Agency for Research on Cancer classified 'shift work that involves circadian disruption' as a probable human carcinogen (Group 2A), on the basis of limited human evidence and sufficient evidence in experimental animals. In this evaluation, a major limitation of the epidemiological studies reviewed was the definition of shift work. A subsequent workshop convened by IARC considered how shift work should be assessed and quantified to improve the validity of future shift work and cancer studies. Since the publication of this working group's recommendations in 2011, the role of strong exposure assessment as a vital component of informative epidemiological studies of shift work has increasingly been recognized. 